\title{
The central region of the Fornax cluster
}

\section{Dwarf galaxies, globular clusters, and cD halo - are there interrelations?}

\author{
M. Hilker ${ }^{1,2}$, L. Infante ${ }^{1}$, and T. Richtler ${ }^{2}$ \\ 1 Departamento de Astronomía y Astrofísica, P. Universidad Católica, Casilla 104, Santiago 22, Chile \\ 2 Sternwarte der Universität Bonn, Auf dem Hügel 71, 53121 Bonn, Germany
}

Received February 8; accepted May 4, 1999

\begin{abstract}
In this paper we briefly review the properties of the dwarf galaxy population at the core of the Fornax cluster, as well as the properties of the extraordinary rich globular cluster system and the $\mathrm{cD}$ halo around the central galaxy NGC 1399. In turn, the question whether a scenario in which dwarf galaxies have been accreted and dissolved in the cluster center can explain the observed properties is addressed. The possibility of accretion of a certain number of dwarf galaxies, the stripping of their globular clusters and gas, and the formation of new globular clusters from stripped infalling gas are discussed. An increase in the specific frequency of the central globular cluster system is only possible, if the infalling gas from stripped dwarfs formed globulars very efficiently and/or accreted and stripped dwarf galaxies possessed a rich globular cluster system themselves. In conclusion, we argue that although the dwarf galaxy infall is a very attractive scenario to explain a number of properties in the system there are probably other physical mechanisms that also take place.
\end{abstract}

Key words: galaxies: clusters: individual: Fornax cluster - galaxies: $\mathrm{cD}$ - galaxies: interaction galaxies: star clusters

\section{Introduction}

The central regions of galaxy clusters are the places with the highest galaxy density in the universe. Dwarf ellipticals $(\mathrm{dE})$ are especially the most strongly clustered types of galaxies in high-density environments (e.g. review by Ferguson \& Binggeli 1994, and references therein). Several striking characteristics are seen in the center region of

Send offprint requests to: M. Hilker

Correspondence to: mhilker@astro.uni-bonn.de clusters: (1) most central galaxies possess extraordinarily rich globular cluster systems (GCS) (see Harris 1991; Richtler 1995 and references therein), but see also apparent counter-examples (see Table 14 in McLaughlin et al. 1994); (2) there often exists a cD galaxy in the center of clusters (e.g. Schombert 1988). (3) different types of dwarf galaxies have different clustering properties (e.g. Vader \& Sandage 1991); (4) in some cases the faint end slope of the dwarf galaxy luminosity function (LF) seems to depend on the cluster-centric distance (e.g. in Coma: Lobo et al. 1997). The question arises on whether these properties may be related through the accretion of dwarf galaxies.

The answer to this question is most probably associated to the formation epoch of galaxy clusters. At that time, it is expected that galaxies were very gas-rich and that interactions between galaxies were more frequent. The number density of galaxies at that epoch in the central region must have been larger than today. Therefore, the initial population of dwarf galaxies played an important role. The favoured theoretical models of galaxy cluster formation predict a steep slope of the initial mass function towards the low-mass end (see a more detailed discussion and references in Sect. 2.1). In contrast, the faint end slope of the observed LF in nearby groups and clusters is significantly flatter (see Ferguson \& Binggeli 1994; Trentham 1998). One possibility that would explain this discrepancy is the accretion and dissolution of dwarf galaxies in cluster centers. It is possible to understand the formation of a rich $\mathrm{GC}$ system and a $\mathrm{cD}$ halo from the infall of gas-poor and gas-rich dwarfs into a dense cluster environment. During the infall of gas-poor as well as gasrich dwarfs in a dense cluster center environment several scenarios are thinkable for forming a rich GCS and a cD halo (see Sect. 5).

Support for such a scenario from the observational side comes from López-Cruz et al. (1997) who compared the properties of clusters with and without a central luminous cD galaxy. They found that clusters without a prominent 
cD galaxy tend to have a steep LF at the faint end and a high fraction of late-type galaxies, and thus seem to be less evolved than clusters with pronounced $\mathrm{cD}$ galaxies and relatively flat $\mathrm{LF}$ at the faint end. They explain this finding by the disruption of dwarf galaxies.

In this study the attention is focused on the properties of the relatively poor, compact, and evolved Fornax cluster, one of the best studied galaxy clusters in the local universe (e.g. Ferguson 1989; Ferguson \& Sandage 1988). Other nearby clusters are believed to be in different evolutionary states. Whereas Virgo (e.g. Sandage et al. 1985; Ferguson \& Sandage 1991) is dominated by late type galaxies and is only half as dense in the center (numbers of galaxies per volume) as Fornax. Centaurus (Jerjen \& Dressler 1997; Stein et al. 1997) and Coma (e.g. Secker \& Harris 1997) show substructures, indicative of a still ongoing dynamical evolution, like for example cluster-cluster or cluster-group merging.

In the first two papers of this series (1998a, 1998b hereafter Paper I and Paper II) we investigated the distribution of galaxies in central Fornax fields. We found two compact objects that belong to the Fornax cluster and might be candidates for isolated nuclei of stripped dwarf ellipticals. However, very few new members were found compared to the study of Ferguson (1989). Thus, the spatial distribution and luminosity function of dwarf galaxies in Fornax (Ferguson \& Sandage 1988) was confirmed.

In this paper we discuss the possibility, whether the infall of dwarf galaxy into the cluster center may play an important role in the enrichment of the central globular cluster system, especially the increase of the globular cluster specific frequency $S_{N}$, as well as the formation of the extended $\mathrm{cD}$ halo.

In the following section we give a compilation of the necessary background of our analysis.

\section{Dwarf galaxies in clusters}

\subsection{Theoretical background on the evolution of dwarf galaxies in clusters}

In their review about dwarf elliptical galaxies, Ferguson \& Binggeli (1994) summarized the formation and evolutionary scenarios that are predicted by theoretical models. It is generally accepted that galaxy formation started from gaseous conditions in the early universe followed by the collapse of primordial density fluctuations, cooling of the gas and subsequent star formation (e.g. White \& Frenk 1991; Blanchard et al. 1992; Cole et al. 1994; Kauffmann et al. 1993; Lacey et al. 1993). In cold dark matter (CDM) dominated models the formation of low-mass galaxies is favored, because for dwarf galaxy halos collapsing at $z \simeq 3-10$ the cooling time is short compared to the freefall time, thus cooling should be very efficient, and accordingly many dwarfs will be formed. A steep slope, $\alpha=-2$, of the initial mass function $\left(N(M) \mathrm{d} M \propto M^{\alpha}\right)$ is predicted (e.g. Blanchard et al. 1992). In contrast, the faint end slope of the observed luminosity functions in nearby clusters are around $\alpha \simeq-1.3 \pm 0.4$ (see Ferguson \& Binggeli 1994). This contradiction is the so-called "overcooling problem" (e.g. Cole 1991). If the CDM model prediction is correct, there must have been active some mechanisms that either counteracted the cooling during the collapse of dwarfs or destroyed the numerous dwarfs after their formation. Plausible mechanisms that involve internal as well as external agents are summarized in the review by Ferguson \& Binggeli (1994).

In the following, we focus our attention on the possibility that many dwarf galaxies have merged with the central galaxy. For a CDM power spectrum in an $\Omega=1$ cosmology the epoch of dwarf galaxy formation is believed to be also the epoch of rapid merging. Kauffmann et al. (1994) included the merging of satellite galaxies in their CDM models and found that most of the observational data can be reproduced when adopting a merging timescale that is a tenth of the tidal friction timescale, and when star formation is suppressed in low-circular-velocity halos until they are accreted into larger systems. Further, efficient merging at all epochs results in a decrease of the faint end slope of the LF compared to the initial predicted value of $\alpha=-2$.

\subsection{Dwarf galaxies and $c D$ halo}

Several authors have suggested that tidal disruption (total dissolution of the galaxy light) of galaxies in cluster centers as well as tidal stripping (only outer parts are affected, a remnant survives) might be related to the formation of $\mathrm{cD}$ halos (see references below). The time of formation is being discussed. Most authors assume that the stripping processes take place after the cluster collapse (e.g. Gallagher \& Ostriker 1972; Richstone 1976; Ostriker \& Hausman 1977; Richstone \& Malumuth 1983). In contrast, Merritt (1984) explained the general appearence of $\mathrm{cD}$ halos as the result of dynamical processes during the cluster collapse. In his scenario the accumulation of slowly-moving galaxies in the cluster core via dynamical friction only plays an important role for groups or clusters with small velocity dispersion $\sigma_{v} \leq 500 \mathrm{~km} \mathrm{~s}^{-1}$ (Fornax: $\sigma_{v} \simeq 360 \mathrm{~km} \mathrm{~s}^{-1}$ ). White (1987) argued that, in the case of tidal disruption and stripping, the distribution of stripped and disrupted material (diffuse light, dark matter, GCs) should be more concentrated to the center than the relaxed galaxy distribution, because galaxies closer to the center are more affected by disruption processes than galaxies outside. In the case of Merritt's model, galaxies formed before the collapse, stripping occured during the collapse, and finally the stripped material is distributed in the same way as the galaxies through collective relaxation.

Furthermore, it is interesting to note that also a large amount of the intracluster gas (seen as X-ray halo) might 
Table 1. Overview of different parameters of Fornax components, as the population of dwarf elliptical and dwarf S0 galaxies, the central GCS, the unresolved stellar light of NGC 1399 and its $\mathrm{cD}$ halo, and the gas component in the central region of the cluster. The assumed distance modulus is $(m-M)_{0}=31.3 \mathrm{mag}$. The profile slope $\alpha$ is the exponent in the density law $\rho \propto r^{\alpha}$, where $\rho$ is either the projected number density or the projected surface brightness profile

\begin{tabular}{|c|c|c|c|c|c|}
\hline $\begin{array}{l}\text { cluster } \\
\text { component }\end{array}$ & $\begin{array}{c}\text { profile slope } \\
\alpha\end{array}$ & $\begin{array}{c}\text { velocity disp. } \\
\sigma_{v} \mathrm{~km} \mathrm{~s}^{-1}\end{array}$ & $\begin{array}{c}\text { metallicity } \\
{[\mathrm{Fe} / \mathrm{H}]}\end{array}$ & $\begin{array}{c}M_{V} \\
{[\mathrm{mag}]}\end{array}$ & $\begin{array}{l}\text { mass } \\
{\left[M_{\odot}\right]}\end{array}$ \\
\hline $\mathrm{dE} / \mathrm{dS}^{a}$ & $-1.0 \pm 0.2$ & 410 to 490 & -1.5 to -0.8 & $-20.8 \pm 0.2$ & \\
\hline $\mathrm{dE} / \mathrm{dS} 0\left(r<0^{\circ} .7\right)$ & $-0.2 \pm 0.1$ & & & $-19.3 \pm 0.4$ & \\
\hline $\mathrm{dE} / \mathrm{dS} 0(r>0.7)$ & $-1.5 \pm 0.3$ & & & $-20.4 \pm 0.3$ & \\
\hline central GCS & $-1.5 \pm 0.2$ & $373 \pm 35$ & -1.8 to 0.3 & $-17.6 \pm 0.2$ & \\
\hline GCS (blue pop.) & $\simeq-1.0 \pm 0.2^{b}$ & $362 \pm 104$ & -1.8 to -0.8 & & \\
\hline GCS (red pop.) & $\simeq-1.7 \pm 0.2^{b}$ & $341 \pm 51$ & -0.9 to 0.3 & & \\
\hline 1399 total light & $-1.6 \pm 0.1$ & & & $-22.33 \pm 0.20^{c}$ & $4.510^{11 d}$ \\
\hline $1399 \mathrm{cD}$ halo & $-1.0 \pm 0.2$ & 270 to $400^{e}$ & $?$ & $-21.65 \pm 0.20$ & $1.910^{10}$ \\
\hline 1399 bulge & $-2.0 \pm 0.2$ & $200($ to 360$)$ & -0.7 to -0.1 & $-21.50 \pm 0.20$ & $1.610^{10}$ \\
\hline $\mathrm{X}$-ray gas $\left(r<0^{\circ} .7\right)$ & $-1.2 \pm 0.1$ & 390 to $460^{f}$ & -0.6 to 0.0 & & $\simeq 2.710^{11 g}$ \\
\hline X-ray gas $\left(r<10^{\prime}\right)$ & & & & & $\simeq 1.210^{10 \mathrm{~g}}$ \\
\hline
\end{tabular}

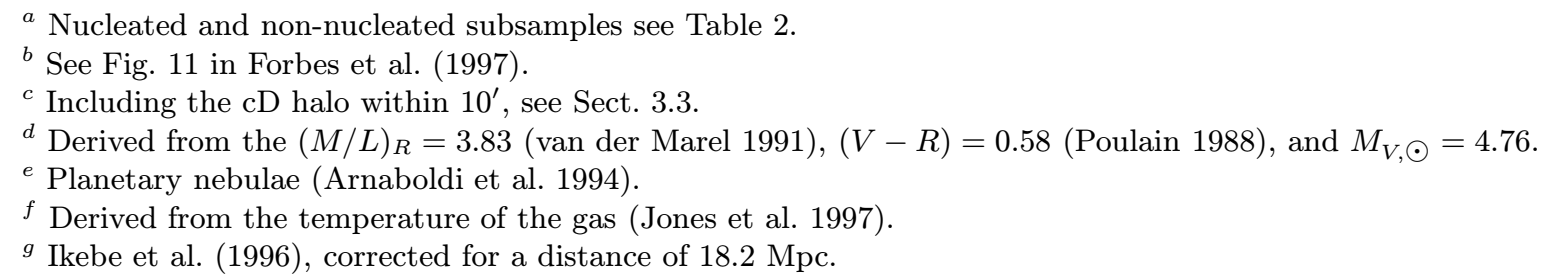

have had its origin in dwarf galaxies, which could have expelled their gas by supernova-driven winds, or stripped off their gas (Trentham 1994; Nath \& Chiba 1995). In the Virgo cluster, for example, Okazaki et al. (1993) estimated that the amount of gas expelled from the E and $\mathrm{S} 0$ galaxies is not adequate to account for the total gas mass in the cluster. Mac Low \& Ferrara (1999) calculated that low mass dwarf galaxies can easily blow away metals from supernovae which might enrich the halo gas.

\section{Properties of dwarf galaxies, GCs, and $\mathrm{cD}$ halo in the Fornax cluster center}

The center of the Fornax cluster hosts the central galaxy NGC 1399 with an extraordinarily rich globular cluster system and an extended $\mathrm{cD}$ halo as well as a halo of $\mathrm{X}$-ray emitting gas. In the following we give a short review on the properties of the different components that have to be considered in the picture of a common evolution. In Table 1 those properties are summarized: the slopes of the surface density profiles, the velocity dispersion, and the ranges of metallicities. Furthermore, the absolute $V$ luminosities and estimated masses are given, if available.

\subsection{Dwarf galaxies in the Fornax cluster}

The most complete investigation of the Fornax dwarf galaxies was done by Ferguson (1989, Fornax Cluster Catalog (FCC)) as well as by Davies et al. (1988, and following papers: Irwin et al. 1990; Evans et al. 1990). As we have shown in Paper I the morphological classification of Fornax members by Ferguson (1989) is very reliable and nearly no $\mathrm{dE}$ has been missed within the survey limits as far as we can judge from the comparison with our sample fields. Thus, the following properties of the Fornax dwarf galaxies are mainly based on the FCC plus the additional new members as presented in Paper I.

The spatial distribution of dEs in Fornax can be represented by a King profile with a core radius of $0.67 \pm$ 0.1 and a center located about $25^{\prime}$ west of NGC 1399 (Ferguson 1989). In order to compare their surface density profile with that of the GCS and the $\mathrm{cD}$ halo light we fitted power laws to the radial distribution of the dEs and dS0s in the extended FCC adopting NGC 1399 as the center. For that we counted galaxies brighter than $B_{\mathrm{T}}=$ $19 \mathrm{mag}$ in 7 equi-distant rings from 0 to $3^{\circ}$. We determined the slopes of the density profiles in the inner $(r<0.8)$ as well as in the outer $\left(0.8<r<3^{\circ}\right)$ part. The dividing radius of 0.8 is about the limit out to where the $\mathrm{cD}$ halo light and the gas envelope have been measured. The results are summarized in Table 2 . In addition, we also give the mean slopes, when fitting a power law to the total profile, and the fitted values for the giant galaxies. The nucleated dwarf galaxies have the steepest slope and are more concentrated towards the central galaxy than the non-nucleated $\mathrm{dE} / \mathrm{dS} 0 \mathrm{~s}$.

The luminosity function (LF) of the Fornax dwarf galaxies was studied by Ferguson \& Sandage (1988) in a region with radius smaller than 2.4 , centered on 
Table 2. Power law slopes of the surface density profiles of Fornax galaxies taken from the FCC (Ferguson 1989)

\begin{tabular}{lccccc}
\hline & all members & all dE/dS0 & nucleated & non-nucl. & E/S0 \\
\hline number & 292 & 192 & 97 & 95 & 27 \\
$r<0.8$ & $-0.30 \pm 0.06$ & $-0.19 \pm 0.03$ & $-0.40 \pm 0.11$ & $-0.20 \pm 0.02$ & $-0.33 \pm 0.04$ \\
$0.8<r<3^{\circ}$ & $-1.51 \pm 0.13$ & $-1.52 \pm 0.28$ & $-2.04 \pm 0.17$ & $-1.06 \pm 0.58$ & $-1.49 \pm 0.56$ \\
total & $-1.04 \pm 0.23$ & $-1.03 \pm 0.26$ & $-1.20 \pm 0.25$ & $-0.86 \pm 0.45$ & $-1.07 \pm 0.24$ \\
\hline
\end{tabular}

Table 3. Faint end slopes of the luminosity function for different subsamples of the Fornax galaxy population

\begin{tabular}{|c|c|c|c|c|}
\hline & $B_{\text {limit }}$ & all members & $\mathrm{dE} / \mathrm{dS} 0$ & non-nucleated \\
\hline$r<3.5$ & 19.5 & $-1.03 \pm 0.09$ & $-0.90 \pm 0.05$ & $-0.73 \pm 0.13$ \\
\hline$r<3.5$ & 19.0 & $-1.23 \pm 0.08$ & $-0.81 \pm 0.07$ & $-0.51 \pm 0.18$ \\
\hline$r<0.7$ & 19.5 & $-1.05 \pm 0.19$ & $-1.27 \pm 0.14$ & $-1.09 \pm 0.30$ \\
\hline$r<0.7$ & 19.0 & $-1.16 \pm 0.25$ & $-1.20 \pm 0.17$ & $-2.01 \pm 0.28$ \\
\hline $0.7<r<2.4$ & 19.5 & $-1.11 \pm 0.07$ & $-0.99 \pm 0.07$ & $-0.95 \pm 0.21$ \\
\hline $0^{\circ} .7<r<2^{\circ} .4$ & 19.0 & $-1.19 \pm 0.09$ & $-0.92 \pm 0.10$ & $-0.63 \pm 0.76$ \\
\hline $\mathrm{F} \& \mathrm{~S} 88^{a}\left(r<2^{\circ} .4\right)$ & 19.5 & $-1.32 \pm 0.09$ & $-1.08 \pm 0.19$ & - \\
\hline
\end{tabular}

${ }^{a}$ Ferguson \& Sandage (1988).

NGC 1399. They found that the nucleated dwarf ellipticals $(\mathrm{dE}, \mathrm{Ns})$ as well as the dwarf lenticular ( $\mathrm{dS} 0)$ galaxies are brighter than the non-nucleated dEs. Further, the faint end slope of the $\mathrm{dE} / \mathrm{dS} 0 \mathrm{LF}$, fitted by a Schechter (1976) function, is quite flat $(\alpha=-1.08 \pm 0.10)$ compared to other clusters like Virgo $(\alpha=-1.31 \pm 0.05)$ or Centaurus $(\alpha=-1.68 \pm 0.56$, Jerjen \& Tammann 1997). Table 3 summarizes the results for the faint end slopes of Schechter function fits to different subsamples of the extended FCC.

Colors and metallicities of dwarf galaxies in Fornax have been studied by photometric as well as by spectroscopic means (e.g. Caldwell \& Bothun 1987; Bothun et al. 1991). Spectroscopically determined metallicities seem to be consistent with the picture that the bluer dwarfs are the more metal-poor ones. The metallicity range for 10 bright $\mathrm{dE}, \mathrm{Ns}$ is $-1.5<[\mathrm{Fe} / \mathrm{H}]<-0.8 \mathrm{dex}$ (Held \& Mould 1994). The metallicities derived from Washington photometric indices for 15 LSB dwarfs are of the same order (Cellone et al. 1996). Concerning ages, all investigated dwarfs possess an old stellar population, some of them a contribution of intermediate-age stars, and only few have signs of recent or ongoing star formation (Held \& Mould 1994; Cellone \& Forte 1996). It seems that the Fornax dEs share the same characteristics as the Local Group dSph population (e.g. review by Grebel 1997).

Radial velocity measurements of 43 Fornax dwarfs $\left(18>B_{\mathrm{t}}>15 \mathrm{mag}\right)$ by Drinkwater et al. (1997) result in a velocity dispersion of $\sigma_{v}=490 \mathrm{~km} \mathrm{~s}^{-1}$, significantly larger than that of 62 giants $\left(B_{\mathrm{t}}<15 \mathrm{mag}\right), \sigma_{v}=$ $310 \mathrm{~km} \mathrm{~s}^{-1}$. According to the authors, this difference cannot be explained by measurement errors.

\subsection{The central globular cluster system}

The globular cluster system of NGC 1399 is one of the best investigated GCSs outside the Local Group. The total number of GCs is about $N_{\mathrm{GC}}=5800 \pm 500$ (Kissler-Patig et al. 1997; Grillmair et al. 1998) within a radius of $10^{\prime}$ from the galaxy center. This is about 10 times the number of GCs in the other Fornax ellipticals, $300 \pm 60<N_{\text {tot }}<700 \pm 100$. Adopting a distance of 18.2 Mpc or $(m-M)_{0}=31.3 \mathrm{mag}$ to NGC 1399 (Kohle et al. 1996, recalibrated with new distances of Galactic GCs, Gratton et al. 1997) the absolute magnitude of NGC 1399 is $M_{V}=-21.75 \mathrm{mag}$ when taking the apparent magnitude values from the literature (Faber et al. 1989, RC3: de Vaucouleurs et al. 1991). This corresponds to a specific frequency of $S_{N}=11.6 \pm 2.0$. If the light of the $\mathrm{cD}$ halo within $10^{\prime}$ is taken into account (see Sect. 3.3), $S_{N}$ is reduced to $6.8 \pm 2.0\left(M_{V \text {,tot }}=\right.$ $-22.33 \mathrm{mag})$. However, distinguishing a $\mathrm{cD}$ halo and a bulge component in the galaxy light, $S_{N}$ for the cD halo would be about $10 \pm 1$ assuming, $S_{N}=3.2$ for the bulge, see Sect. 9, the average value of the other early-type Fornax galaxies (Kissler-Patig et al. 1997). Thus, the building up of the GCS of the $\mathrm{cD}$ halo component must have been very efficient.

The color distribution of the GCs around NGC 1399 is very broad compared to most other GCSs in Fornax ellipticals and can only be explained by a multimodal or perhaps just a bimodal GC population (e.g. Ostrov et al. 1993; Kissler-Patig et al. 1997, and Forbes et al. 1997). Spectroscopic analysis of 18 GCs by Kissler-Patig et al. (1998) shows a metallicity range between -1.6 and -0.3 dex (with possible peaks at -1.3 and -0.6 dex), and two exceptional GCs at about 0.2 dex, located in the red (metal rich) tail of the color distributions. 
The comparison of the line indices with theoretical evolutionary models suggests that most of the GCs are older than at least $8 \mathrm{Gyr}$. If one fits the GC color distribution with two Gaussians, the number ratio of metal rich (red) to metal poor (blue) GCs is about 1:1 (Forbes et al. 1997).

The radial extension of the GCS around NGC 1399 can be traced out to about $10^{\prime}(\simeq 53 \mathrm{kpc})$. The slope of the GC surface density profile, $\rho \propto r^{\alpha}$, is about $\alpha=-1.5 \pm 0.2$, when taking the average of the published values. (Forbes et al. 1997) found that the distribution of the blue GC subpopulation is even flatter $(\alpha \simeq-1.0 \pm 0.2)$, whereas the red GCs are more centrally concentrated $(\alpha \simeq-1.7 \pm 0.2)$, comparable to the slope of the galaxy light $(\alpha=-1.6 \pm 0.1)$. See Fig. 2 for a schematic overview.

Radial velocities of 74 GCs around NGC 1399 have been measured (Kissler-Patig et al. 1999; Minniti et al. 1998; Kissler-Patig et al. 1998). The velocity dispersion for the whole sample is $\sigma_{v}=373 \pm 35 \mathrm{~km} \mathrm{~s}^{-1}$. No differences can be seen between the red and blue subpopulations. However, there exists a radial dependence of the velocity dispersion in the sense that $\sigma_{v}$ rises from $263 \pm 92$ to $408 \pm 107 \mathrm{~km} \mathrm{~s}^{-1}$ between $2^{\prime}$ and $8^{\prime}$ (Kissler-Patig et al. 1999).

\section{3. $c D$ halo and bulge}

The galaxy light of NGC 1399 follows an extended cD profile (Schombert 1986; Killeen \& Bicknell 1988) out to a radial distance of about 34 arcmin from the galaxy center $\left(\Sigma_{B}=28 \mathrm{mag}\right.$ isophotal surface brightness level). This is about $180 \mathrm{kpc}$ in Fornax distance $(18.2 \mathrm{Mpc})$ and comparable to the extent of the X-ray envelope (Ikebe et al. 1996; Jones et al. 1997).

The determination of the stellar population parameters of the outer $\mathrm{cD}$ halo, like accurate photometric colors, metallicity or velocity dispersion, is very difficult due to the low surface brightness. Long slit spectra have been taken for the stellar bulge population within a radius of about 1'5 from the center of NGC 1399 (Franx et al. 1989; Bicknell et al. 1989). The velocity dispersion is about $200 \mathrm{~km} \mathrm{~s}^{-1}$ at 1.5 and rises within the central $10^{\prime \prime}$ to a central value of about $360 \mathrm{~km} \mathrm{~s}^{-1}$. Besides the GCS, a useful tracer for the stellar population at larger radii is the population of planetary nebulae ( $\mathrm{PNe})$. Arnaboldi et al. (1994) studied the kinematics of $37 \mathrm{PNe}$ out to a radius of 4.5 . They found an increase in the velocity dispersion with increasing radius from $269 \mathrm{~km} \mathrm{~s}^{-1}$ for $r<2$ ! 6 to $405 \mathrm{~km} \mathrm{~s}^{-1}$ for $2^{\prime} .6<r<4.5$ (18 of the $37 \mathrm{PNe}$ ).

\subsubsection{Luminosity and surface brightness profile}

In this subsection we divide the light profile of NGC 1399 into a $\mathrm{cD}$ halo and a bulge component in order to compare
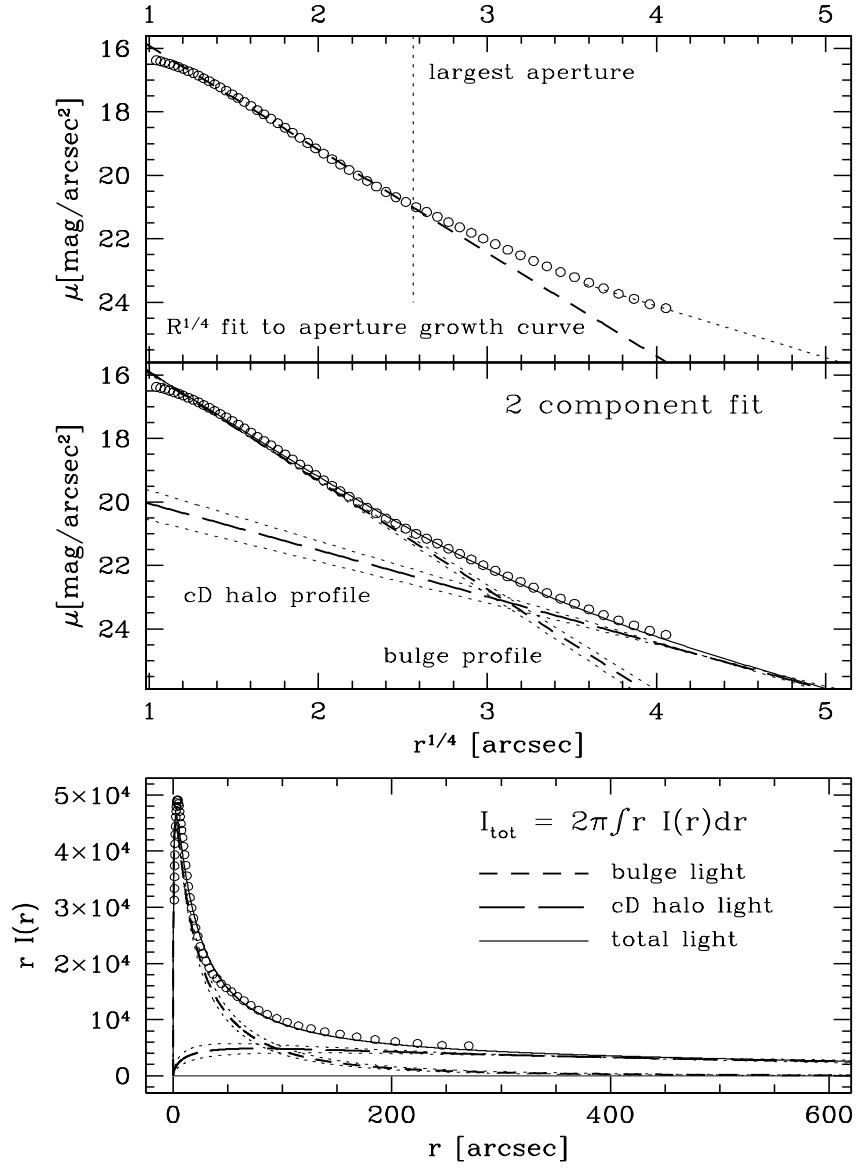

Fig. 1. The upper two panels show the surface brightness profile (open circles) of NGC 1399 plotted versus $r^{1 / 4}$. The change of the slope to a flat $\mathrm{cD}$ halo is clearly visible. In the uppermost panel, the dashed line corresponds to the $R^{1 / 4}$ law extrapolation of an aperture growth curve used to derive the apparent magnitude $V=9.55$ mag (Faber et al. 1989, RC3). The largest aperture given by Burstein et al. (1984) is indicated. The dotted extension of the surface brightness profile at the faint end represents the slope between $2^{\prime}$ and $10^{\prime}$ by Killeen \& Bicknell (1988). The dashed lines in the second panel represent the single components of the $\mathrm{cD}$ halo and the underlying bulge light, when fitting the sum of two de Vaucouleurs laws (solid line). The dotted lines give the ranges for possible fits. In the lowermost panel, $r I(r)$ is plotted versus the radial distance to the center of NGC 1399. Again, the dashed curves describe the single components and the dotted ones the possible ranges. Within $10^{\prime}$ the total luminosity $I_{\text {tot }}$ of the $\mathrm{cD}$ halo light is slightly higher than that of the bulge light

their characteristics with those of the GCS and the dwarf galaxy population. We determined the absolute luminosity of the $\mathrm{cD}$ halo in the following way: in the $\mu-r^{1 / 4}$ plot (Fig. 1, upper panels) one can see that the SB profile of NGC 1399 (determined from the NE CCD field F2) changes its slope at about $50^{\prime \prime}$. We fitted the total profile 
by the sum of two de Vaucouleurs laws:

$\mu(r)=Z P_{\text {cal }}-2.5 \cdot \log \left[I_{\text {gal }}^{0} \cdot \exp \left(-\left(r / \alpha_{\text {gal }}\right)^{1 / 4}\right)+I_{\text {cD }}^{0}\right.$. $\left.\exp \left(-\left(r / \alpha_{\mathrm{cD}}\right)^{1 / 4}\right)\right]$.

The steeper, more concentrated profile represents the luminosity of the bulge without $\mathrm{cD}$ halo, whereas the flatter, more extended profile contains the light of the $\mathrm{cD}$ halo. The total luminosity of each component is $I_{\text {gal,cD }}^{\text {tot }}=$ $2 \pi \int r I_{\text {gal }, \mathrm{cD}}(r) \mathrm{d} r$. We restricted our calculations to within a radius of $10^{\prime}$ where the number of detected GCs fades into the background. The dashed lines in Fig. 1 represent the "best" fit (data points inside 1". 5 radius have been omitted). The dotted lines give the ranges for possible fits. The surface density slopes of both profiles are $\alpha=$ $-2.0 \pm 0.2$ and $\alpha=-1.0 \pm 0.2$ respectively, if $\Sigma(r) \propto r^{\alpha}$. The slope of the combined profile is $\alpha=-1.6 \pm 0.1$ (see also Fig. 2).

In the literature one finds an apparent magnitude for NGC 1399 of $V=9.55 \mathrm{mag}$ (Faber et al. 1989, RC3: de Vaucouleurs et al. 1991, adopting a mean $(B-V)$ color of $1.0 \mathrm{mag}$, Goudfrooij et al. 1994). This magnitude is derived from an aperture growth curve extrapolation. with a maximum aperture of diameter 1!.5 (Burstein et al. 1984). A 1-component fit of an $R^{1 / 4}$ law within 1.5 (the largest aperture in Burstein et al. 1984) is shown in Fig. 1 (uppermost panel). Adopting an absolute magnitude of $M_{V}=$ $-21.75 \mathrm{mag}$ for the integrated light under this profile, the total luminosity of the bulge light from the 2-component fit (middle panel) is $M_{V \text {,bulge }}=-21.50 \pm 0.20 \mathrm{mag}$ (about $80 \%$ of the luminosity given in the literature). The luminosity for the $\mathrm{cD}$ halo is $M_{V, \mathrm{cD}}=-21.65 \pm 0.2 \mathrm{mag}$ and for the whole system within $10^{\prime} M_{V \text {,tot }}=-22.33 \pm$ $0.2 \mathrm{mag}$.

Another check for the correct proportion of the luminosities of the different components can be made by comparing the integrated flux within an aperture of 1.5 with the total flux within $10^{\prime}$. Adopting $V=10.30 \mathrm{mag}$ for the 1.5 aperture (Burstein et al. 1984), we derive $M_{V, \text { tot }}=-22.27 \pm 0.2 \mathrm{mag}$ for the whole system, in excellent agreement with the value given above.

Note that the total luminosity of the $\mathrm{cD}$ halo is about 180 times the luminosity of a typical dwarf galaxy with $M_{V}=-16.0 \mathrm{mag}$ or 2.2 times the total luminosity of the present dEs and dS0s in Fornax.

\section{Comparison of corresponding properties}

In the previous section we presented the properties of the different components of NGC 1399 and the galaxy population in the center of the cluster. In Fig. 2 we give a schematic overview of the surface density/brightness (SD/SB) profiles of the different components and their extension. The profiles are arbitrarily shifted in the ordinate axis. The surface densities of GCs and galaxies are number

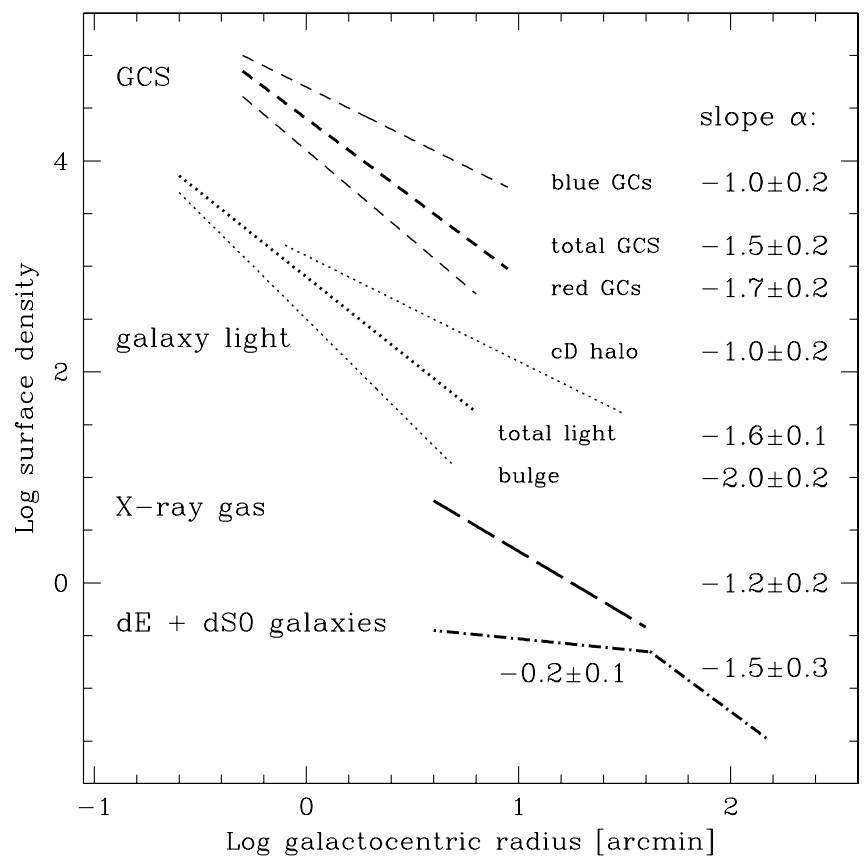

Fig. 2. Schematic overview of the surface density or brightness profiles of different subpopulations in the Fornax cluster and their extensions. The profiles are arbitrarily shifted in the ordinate axis. Also the units are arbitrarily chosen. The profiles of the GCs and galaxies are surface density profiles, the one of the galaxy light is a surface brightness profile, and the one of the X-ray gas is again a particle (number) density profile (see text for more details). The slopes $\alpha\left(\rho \propto r^{\alpha}\right)$ and their uncertainties are indicated on the right hand side

densities, whereas the profile of the galaxy light is a surface brightness profile. Nevertheless, this is comparable to the number density profile, if one assumes similar stellar populations and, accordingly, similar $M / L_{V}$ ratios. The surface density of the X-ray gas is again a particle (number) density, $n_{\text {gas }} \propto r^{\gamma}$. The gas density profile is derived from the surface brightness distribution of the X-ray gas $S(r) \propto r^{\tau}$ under the assumption of isothermal conditions $(\tau=(\gamma+1) / 2)$ for a radius larger than about $10 \mathrm{kpc}$ (Jones et al. 1997).

The plot shows that the profile slopes of the blue GC population and the $\mathrm{cD}$ halo light are strikingly similar, whereas the distribution of $\mathrm{dE} / \mathrm{dS} 0$ galaxies is somewhat flatter in the central 0.7 and slightly steeper outside. Interestingly, the surface density profile of the X-ray gas is also very similar in slope and extension to the $\mathrm{cD}$ halo light and the blue GCs. In contrast, the profile of the bulge light of NGC 1399 is significantly steeper than all other profiles.

The same behaviour can be seen in the velocity dispersion. It is comparable for dwarf galaxies and GCS, whereas the stars in the stellar bulge have a lower $\sigma_{v}$ (see also Minniti et al. 1998; Kissler-Patig et al. 1999). This agreement in morphological and dynamical properties of GCs, the $\mathrm{cD}$ halo light, and perhaps also the gas particles might 
suggest that these components share a common history (or origin). In the next section we describe some scenarios that might have happened when dwarf galaxies interacted with the central cluster galaxy.

\section{Disruption, accretion and stripping of dwarf galaxies}

What are the possible consequences, when dwarf galaxies of different types interact with the central galaxy, especially with respect to the formation of a $\mathrm{cD}$ halo and $\mathrm{a}$ rich GCS?

We make a distinction between two main cases:

(1) the infall of gas-poor dwarfs, for example dwarf ellipticals, where only the existing stellar component is involved in the interaction process, and

(2) the infall of gas-rich dwarfs, where the interaction of the gas has to be considered and might play an important role in the formation of new stellar populations.

A further sub-division of these cases is:

(a) the dwarf galaxy will be totally dissolved in the interaction process,

(b) only parts of the dwarf galaxy (for example gas and/or globular clusters) will be stripped during the passage through the central cluster region,

(c) the dwarf galaxy neither loses gas nor stars nor clusters to the cluster center, but might change its morphological shape because of tidal interactions (for example getting more compact or splitting into two).

In the next subsections we discuss the possible consequences for the different cases.

\subsection{Gas-poor dwarfs}

(1a): in this case the stellar population of the dwarf galaxy will be disrupted in tidal tails and the stellar light will be smeared out in the potential well of the cluster center. Most affected by this process are the faintest dEs (or dSphs, Thompson \& Gregory 1993). In clusters with a low velocity dispersion or at the bottom of a local potential well in a rich cluster (Zabludoff et al. 1990), the light of several dissolved dwarfs may form an extended, diffuse $\mathrm{cD}$ halo. Existing GCs of the dwarfs will survive and contribute to the central GCS. In the Local Group, an example for this scenario may be the Sagittarius dSph which is dissolving into our Galaxy, adding 4 new GCs to the GCS of the Milky Way (Da Costa \& Armandroff 1995). However, only few dwarf galaxies with a very rich GCS compared to their luminosity are known (Miller et al. 1998; Durrell et al. 1996). In Sect. 6 we estimate under which conditions the accretion of gas-poor dwarfs and their GCS can increase $S_{N}$ of a central GCS.

Finally, the nuclei of dE,Ns can survive the dissolution of their parent galaxy and may appear as GCs (Zinnecker et al. 1988; Bassino et al. 1994). The nuclear magnitudes of all Virgo dE,Ns (Binggeli \& Cameron 1991), for example, fall in the magnitude - surface brightness sequence is defined by the GCs (e.g. Binggeli 1994).

(1b): like in the case 1a the stripped stars and GCs will be distributed around the central galaxy. In this case the question arises on how large the number of stripped GCs is compared to the luminosity of the stripped stellar light. If GCs could be stripped from regions with a high local $S_{N}$, this would also increase $S_{N}$ of the central GCS. According to model calculations by Muzzio et al. (1984, see also review by Muzzio 1987), the tidal accretion of GCs and stars can be an important process in the dynamical evolution of GCSs in galaxy clusters.

In some galaxies the GCS is more extended than the underlying stellar light, which has the consequence that the local $S_{N}$ increases with galactocentric distance; for example NGC 4472 has a global $S_{N}$ of 5.5 and a local $S_{N}$ larger than 30 at $90 \mathrm{kpc}$ (McLaughlin et al. 1994). Forbes et al. (1997) and Kissler-Patig et al. (1999) suggest that the stripping of the outermost GCs and stars from such a galaxy naturally increases the $S_{N}$ of the central GCS. It would be interesting to investigate whether this is also true for the GCSs of dwarf galaxies.

Furthermore, it would be interesting to know how the tidal stripping process changes the shape of the remaining galaxy. Kroupa (1997) simulated the interaction of a spherical low-mass galaxy with a massive galactic halo and found that the model remnants share the properties of dwarf spheroidals. On the other hand, M 32 may be an example for a tidally compressed remnant, whose GCs have been stripped (e.g. Faber 1973; Cepa \& Beckman 1988).

(1c): in this case the dwarf galaxy does not contribute to the formation of $\mathrm{cD}$ halo and central GCS. However, as in $1 \mathrm{~b}$ one might speculate about the change of the morphological shape after a passage of the galaxy through the cluster center.

Note that, except in their nuclei, the metallicity of GCs in dEs as well as the metallicity of the bulk of their stars is very low $(-2.5<[\mathrm{Fe} / \mathrm{H}]<-1.0 \mathrm{dex}$, see the review on Local Group dwarfs by Hodge 1994). Therefore, stripped GCs from these galaxies will only contribute to the metalpoor population of the central GCS. And accordingly, the $\mathrm{cD}$ halo should have quite a blue color.

\subsection{Gas-rich dwarfs}

(2a): for the stellar population and GCs see 1a. The infalling gas will experience the thermal pressure of the hot medium in the central galaxy. The densities can be high enough that star formation occurs and the formation of many dense and compact star clusters is possible (e.g. Ferland et al. 1994). As mentioned in Sect. 3.4, stripped gas that was not converted into stars may contribute to the intracluster X-ray gas in the cluster center 
Table 4. Initial conditions for GCSs of dwarf galaxies in the Monte Carlo simulations. $N_{\mathrm{gc}}$ is the possible number of GCs in the magnitude bin. $S_{N}$ gives the range of specific frequencies that can be achieved with the number of GCs

\begin{tabular}{lcccccccc}
\hline bin & $<-15.5$ & $-15.5:-14.5$ & $-14.5:-13.5$ & $-13.5:-12.5$ & $-12.5:-11.5$ & $-11.5:-10.5$ & $-10.5:-9.5$ & $-9.5:-8.5$ \\
\hline$N_{\mathrm{gc}}$ & & $6-20$ & $2-9$ & $1-5$ & $0-2$ & $0-1$ & $0-1$ & $0-1$ \\
$S_{N}$ & 4.5 & $1.8-16.6$ & $1.7-18.8$ & $2.1-28.8$ & $0-29$ & $0-36$ & $0-91$ & $0-251$ \\
\hline
\end{tabular}

(see Nath \& Chiba 1995). The open questions here are, how many "new" star clusters will survive the further cluster center evolution, and how large the number of surviving clusters is compared to the light of newly formed stars which contribute to the total light of the central galaxy and/or cD halo. In other words, it is unclear whether the formation of new GCs is so efficient that it can increase $S_{N}$ of the central GCS.

Some constraints/estimations that can be made from observations of very young star clusters in merging galaxies and starburst galaxies are presented in Sect. 7.

(2b): the stripping of a gas-rich galaxy mainly affects the gas component that may form new stars and clusters as mentioned in case 2a. Nulsen (1982, see also Ferguson \& Binggeli 1994) estimated a typical mass loss rate from infalling dwarfs of

$$
\dot{M}=7.410^{-2} M_{\odot} \mathrm{yr}^{-1} n r_{\mathrm{kpc}}^{2} \sigma_{\mathrm{km} \mathrm{s}^{-1}}
$$

where $n$ is the gas density in the cluster, $r_{\mathrm{kpc}}$ the dwarf galaxy radius in $\mathrm{kpc}$, and $\sigma_{\mathrm{km} \mathrm{s}^{-1}}$ the velocity dispersion of the cluster. A stripping time scale for a typical dwarf irregular (dI), $r=4 \mathrm{kpc}$, gas mass $M_{\text {gas }}=10^{8} M_{\odot}$, and $\sigma=400 \mathrm{~km} \mathrm{~s}^{-1}$ (Fornax) is $t_{\mathrm{S}}=0.5 \mathrm{Gyr}$, when adopting $n=8.210^{8} \mathrm{~cm}^{-3}$ for the central gas density of the Fornax cluster (Ikebe et al. 1996). The fact that dEs are more concentrated towards cluster centers than dIs is interpreted as a result of this stripping scenario (dEs being the remnants of stripped dIs, e.g. Lin \& Faber 1983; Kormendy 1985). Furthermore, non-nucleated dEs have a quite low GC $S_{N}$ in contrast to dE,Ns, for which $S_{N}$ increases with decreasing luminosity (Miller et al. 1998). One might speculate that not only gas but also GCs have been stripped, whereas the $\mathrm{dE}, \mathrm{Ns}$ have a different evolution history.

(2c): the passage of a gas-rich dwarf through the intergalactic gas in a cluster might trigger star formation in the dwarf galaxy itself (Silk et al. 1987) and might enrich its GCS (see Sect. 7). Ferguson \& Binggeli (1994) suggest that a galaxy falling into the cluster for the first time in the present epoch encounters such high densities that stars can form long before ram pressure becomes efficient. A further close passage then can result in the cases $2 \mathrm{a}$ or $2 b$.

In all these cases there are no restrictions for the metallicity of newly formed GCs and stars. Their metallicity depends on the gas enrichment history of the accreted or stripped galaxies themselves. Of course, a lower limit is the metallicity of the interstellar matter of the dwarf galaxy. As an estimation for this lower limit can serve the metallicity of the young populations in the Local Group dSphs and irregulars. It seems that the secondary stellar population has metallicities more metal-rich than $[\mathrm{Fe} / \mathrm{H}]=-1.2$ dex, in some cases even up to solar values (e.g. Grebel 1997).

\section{Enhancement of $S_{N}$ by accretion of gas-free dwarf galaxies}

The possibility that the accretion of gas-poor dwarfs can increase $S_{N}$ of the central GCS requires that the $S_{N}$ value of a large number of accreted dwarfs themselves is very high. Only few examples of dwarf galaxies with very high GC frequencies are known. In the Local Group the Fornax and Sagittarius dwarf spheroidals have extraordinarily high $S_{N}$ values: $29 \pm 6$ and $25 \pm 9$ respectively (Durrell et al. 1996). Their absolute luminosities are about $M_{V}=-13$ mag. Durrell et al. (1996) found in the Virgo cluster two dE,Ns fainter than $M_{V}=-15.5$ mag that have GC specific frequencies in the order of $S_{N}=14 \pm 8$. Recently Miller et al. (1998) found that exclusively dE,Ns can possess high $S_{N}$ GCSs, whereas dEs have "normal" values. In this respect, it is worthwhile noting that the nuclei of $\mathrm{dE}, \mathrm{N}_{\mathrm{s}}$ could be merged globular clusters, and thus the $S_{N}$ of these galaxies might have been even higher in the past. It seems that all of the high $S_{N}$ dwarf galaxies belong to the faint luminosity end of the dwarf galaxy population. Thus, their total numbers of GCs are very small, $4<N_{\text {tot }}<20$ and it might reflect the stochastic effect, where a low mass dwarf is able to produce no, 1, 2, or several clusters.

We tested with Monte Carlo (MC) simulations the possibility that 2500 GCs were captured by gas-poor dwarf galaxy accretion in the center of the Fornax cluster. The number 2500 comprises about the blue GC subpopulation. We assumed that galaxies with absolute luminosities in the range $-18.0<M_{B}<-8.5$ mag have been accreted. Each galaxy contains GCs according to its luminosity. For galaxies with $-18.0<M_{B}<-15.5$ we adopted a mean GC specific frequency of $S_{N}=4.5$ (Durrell et al. 1996). In the fainter magnitude bins, the number of GCs was chosen randomly in such a way that the ranges of observed $S_{N}$ (Miller et al. 1998) were reproduced. In Table 4 the initial conditions for the dwarf GCSs are summarized. We simulated 3 cases, a very optimistic one (simulation run 1 , very faint dwarfs can also possess GCs), a pessimistic case (run 3), where no dwarf fainter than $M_{B}=-12.5$ can possess GCs (as it seems to be the case for the Local Group dSphs), and a medium case (run 2, dwarfs fainter then 


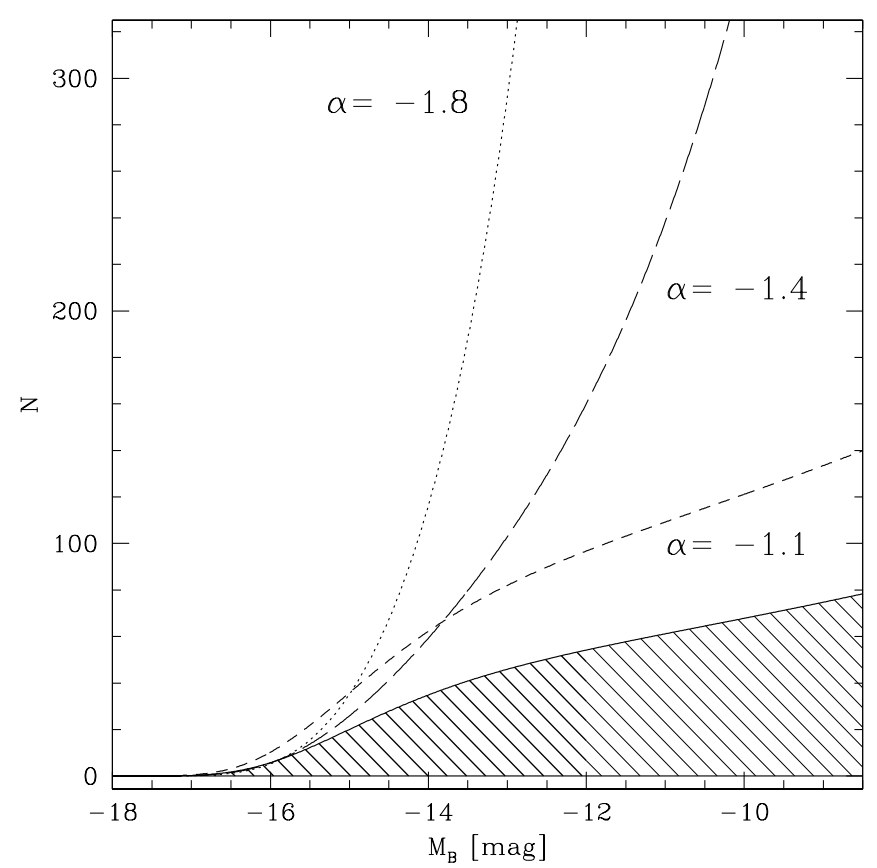

Fig. 3. The dotted and dashed lines are initial Schechter-type luminosity functions with different slopes as indicated. The hatched area represents the present day luminosity function for $\mathrm{dE}$ and dS0 galaxies in Fornax. Note that for $M_{B}>$ -12.0 mag the galaxy counts in Fornax are incomplete

$M_{B}=-10.5$ can not possess any GC). However, if faint $\mathrm{dEs}$ are already stripped dwarf galaxies, the simulation run 1 or 2 seems more reasonable.

We started our simulations with an initial Schechtertype LF with a given characteristic luminosity $M^{*}$ and faint end slope $\alpha$. Then we "disrupted" galaxies of randomly chosen luminosities as long as 2500 GCs have been accumulated, considering the condition that the final LF resembles the present one of the Fornax cluster. We have chosen the following initial faint end slopes: $\alpha=-1.1$ (the present day faint end slope of $\mathrm{dE} / \mathrm{dS} 0 \mathrm{~s}$ ), $\alpha=-1.4$, and $\alpha=-1.8$. We varied the characteristic luminosity between $M^{*}=-15.3$ (present day), $M^{*}=-16.3$, and $M^{*}=-17.3$. The brighter $M^{*}$ the higher is the fraction of disrupted dwarf galaxies at the bright end of the LF. Figure 3 shows the initial LFs with different slopes compared to the present day LF (hatched area). For each simulation we calculated the total number of disrupted dwarfs $N_{\text {tot }}$, their total luminosity $M_{B \text {,tot }}$, the fraction of their light compared to the $\mathrm{cD}$ halo light $\Delta L=L_{\mathrm{dw}} / L_{\mathrm{cD}}$, and the specific frequency of GCs compared to the disrupted stellar light $S_{N, \mathrm{dw}}$. In addition, we estimated the mean metallicity of the accreted GCs. For galaxies brighter than $M_{V}=-13 \mathrm{mag}$, we adopted the metallicity-luminosity relation given by Côté et al. (1998), $\overline{[\mathrm{Fe} / \mathrm{H}]}=2.31+0.638 \cdot M_{V}+0.0247 \cdot M_{V}^{2}$. For the fainter dwarfs, a metallicity-luminosity relation was derived from a linear regression to the Côté et al. data with $M_{V}<-13$ mag, $\overline{[\mathrm{Fe} / \mathrm{H}]}=-0.10 \cdot M_{V}-3.13$. With this relation, the
Table 5. Results of MC simulations for the disruption of gaspoor dwarfs in the center of the Fornax cluster. In simulation run 1 all dwarfs down to $M_{B}=-8.5 \mathrm{mag}$ can possess GCs (see also Table 4), whereas in run 2 and 3 only dwarfs brighter than $M_{B}=-10.5$ and $-12.5 \mathrm{mag}$ can possess GCs, respectively. $\alpha$ and $M^{*}$ are the parameters for the initial LF, $N_{\text {tot }}$ is the number of galaxies that has been disrupted to account for $2500 \mathrm{GCs}, S_{N, \mathrm{dw}}$ is the specific frequency of captured GCs compared to the disrupted galaxy light, $\Delta L=L_{\mathrm{dw}} / L_{\mathrm{cD}}$ is the ratio of disrupted galaxy light $M_{B, \mathrm{t}}$ and the present day $\mathrm{cD}$ halo light, and $\overline{[\mathrm{Fe} / \mathrm{H}]}$ is the mean metallicity of the accreted GCs

\begin{tabular}{rrrrrrrr}
\hline Run & $\alpha$ & $M^{*}$ & $N_{\text {tot }}$ & $M_{B, \mathrm{t}}$ & $S_{N, \mathrm{dw}}$ & $\Delta L$ & $\overline{[\mathrm{Fe} / \mathrm{H}]}$ \\
\hline 1 & -1.1 & -15.3 & 1000 & -20.6 & 7.1 & 1.0 & -1.7 \\
& -1.1 & -16.3 & 450 & -20.9 & 5.5 & 1.3 & -1.5 \\
& -1.1 & -17.3 & 260 & -20.9 & 5.0 & 1.3 & -1.3 \\
& -1.4 & -15.3 & 3180 & -20.0 & 15.0 & 0.5 & -2.0 \\
& -1.4 & -16.3 & 1630 & -20.6 & 7.7 & 1.0 & -1.7 \\
& -1.4 & -17.3 & 950 & -20.8 & 5.9 & 1.1 & -1.5 \\
& -1.8 & -15.3 & 4050 & -19.6 & 22.3 & 0.4 & -2.1 \\
& -1.8 & -16.3 & 3490 & -19.9 & 15.7 & 0.5 & -2.0 \\
& -1.8 & -17.3 & 3060 & -20.1 & 12.4 & 0.6 & -1.9 \\
2 & -1.1 & -15.3 & 1190 & -20.7 & 6.7 & 1.0 & -1.7 \\
& -1.1 & -16.3 & 513 & -20.9 & 5.3 & 1.3 & -1.5 \\
& -1.1 & -17.3 & 248 & -20.9 & 4.9 & 1.3 & -1.3 \\
& -1.4 & -15.3 & 6330 & -20.5 & 9.5 & 0.9 & -1.9 \\
& -1.4 & -16.3 & 2340 & -20.7 & 6.4 & 1.0 & -1.6 \\
& -1.4 & -17.3 & 1240 & -20.8 & 5.6 & 1.1 & -1.5 \\
& -1.8 & -15.3 & 13770 & -20.5 & 9.2 & 0.9 & -1.9 \\
& -1.8 & -16.3 & 9670 & -20.6 & 7.9 & 1.0 & -1.8 \\
& -1.8 & -17.3 & 7530 & -20.7 & 7.1 & 1.0 & -1.7 \\
3 & -1.1 & -15.3 & 1200 & -20.8 & 5.9 & 1.1 & -1.6 \\
& -1.1 & -16.3 & 480 & -20.9 & 5.1 & 1.3 & -1.5 \\
& -1.1 & -17.3 & 270 & -21.0 & 4.8 & 1.4 & -1.3 \\
& -1.4 & -15.3 & 9020 & -21.1 & 5.2 & 1.5 & -1.8 \\
& -1.4 & -16.3 & 2380 & -21.0 & 5.2 & 1.4 & -1.6 \\
& -1.4 & -17.3 & 1150 & -21.0 & 4.9 & 1.4 & -1.4 \\
& -1.8 & -15.3 & 23810 & -21.5 & 3.8 & 2.2 & -1.8 \\
& -1.8 & -16.3 & 11740 & -21.2 & 4.6 & 1.7 & -1.7 \\
-1.8 & -17.3 & 8250 & -21.1 & 4.7 & 1.5 & -1.6 \\
\hline & & & & & & & \\
& & &
\end{tabular}

GCs of the faintest dwarfs in our simulations, $M_{B}=-8.5$ mag, have a mean metallicity of about $\overline{[\mathrm{Fe} / \mathrm{H}]}=-2.3$ dex. Table 5 summarizes the results of our simulations.

The MC simulations show that high $S_{N}$ values around 10 can only be achieved under the assumption that dwarf galaxies fainter than $M_{B}=-10.5 \mathrm{mag}$ can possess at least one GC and that the faint end slope of the initial LF is at least as steep as $\alpha=-1.4$. The dissolved light then comprises about $60-90 \%$ of the present day $\mathrm{cD}$ halo light (within 10'). However, the number of dissolved galaxies in these cases, $3000-14000$, is very high. It has to be shown 
whether theoretical simulations of cluster evolution can reproduce such high destruction rates, when including also very low mass dwarf galaxies. Moreover, the mean metallicity of the accreted GCs for the cases with high $S_{N}$ values would be about 0.5 dex lower than the observed metalpoor peak at -1.3 dex. In Sect. 9 we discuss, whether the mixture of the presented accretion process with stripping of GCs and new formation from infalling gas can explain the luminosity of the $\mathrm{cD}$ halo together with the observed $S_{N}$ of the central GCS in the Fornax cluster.

\section{Efficiency of new cluster formation by the accretion of gas-rich dwarf galaxies}

In this section we assume that the gas of stripped dwarfs forms stars and clusters with the same proportion as has been determined for merging and starburst galaxies. Many examples for young GC candidates in mergers are known: e.g. NGC 3597 (Lutz 1991), NGC 1275 (Holtzman et al. 1992; Nørgaard-Nielsen et al. 1993; NGC 5018 (Hilker \& Kissler-Patig 1996), NGC 7252 (Whitmore et al. 1993; Schweizer \& Seitzer 1993; Whitmore \& Schweizer 1995). The number of newly formed clusters differs from case to case and seems to depend on the amount of gas that is involved in the merging process. The precondition that is needed to form a bound cluster is a cold gas cloud with high density in its core (e.g. Larson 1993). Furthermore, the local star formation efficiency has to be very high and has to occur on a short timescale in order to avoid an early disruption by strong stellar winds and by supernova explosions of the most massive stars (Brown et al. 1995; Fritze-v. Alvensleben \& Burkert 1995). The best candidates for the progenitors of the clusters are the massive, embedded cores of (super) giant molecular clouds (e.g. Ashman \& Zepf 1992; Harris \& Pudritz 1994). Elmegreen et al. (1993) have shown that large molecular cloud complexes can form in interacting systems. The high densities in the cores that are necessary for the cluster collapse can be induced by direct cloud-cloud collision as well as by an increase of the ambient gas pressure as a result of a merger (Jog \& Solomon 1992). Furthermore, the high velocities of colliding gas during mergers might act as dynamical heating that counteracts a fast cooling which would prevent an efficient cluster formation. In this way metal-rich gas, where the cooling times are normally short, could also efficiently form GCs.

What is the observed cluster formation efficiency in merger and starburst galaxies? Meurer et al. (1995) investigated the ultraviolet (UV) properties of young clusters in nine starburst galaxies (blue compact dwarfs as well as ultraluminous mergers). On average, about $20 \%$ of the UV luminosity comes from clusters. But is this percentage sufficient to increase the specific frequency of the GCS? Before answering this question one has to know how many young clusters will survive the evolution of several Gyr.
Fritze v. Alvensleben \& Kurth (1997) calculated with the help of stellar population evolutionary models (Fritze v. Alvensleben \& Burkert 1995) that the young Antennae (NGC 7252) clusters will evolve into a typical GCS. However, they do not exclude the possibility that up to $60 \%$ of the present clusters may be destroyed by dynamical effects during the evolution of the cluster system. This value is the result of semi-analytical model calculations by Vesperini (1997), who simulated the evolution of a original GC population in a spiral. Note that most of the destroyed clusters are low mass clusters. Thus the destroyed cluster mass is a much smaller percentage of the initial total cluster mass. On the other hand, Okazaki \& Tosa (1993) estimated that about $60 \%$ in mass of an initial GC population, whose initial mass function $\phi$ is approximated by the power law $\phi=\mathrm{d} N / \mathrm{d} M \propto M^{-\alpha}$, with $\alpha \simeq 2$, will be destroyed after evolving into the present GCLF. In the following we will assume that a dissolution of 20 to $60 \%$ of the cluster mass or light is reasonable.

\subsection{Increase of $S_{N}$ in a starburst galaxy}

Coming back to the question, whether a strong starburst like those investigated by Meurer et al. (1995) can increase $S_{N}$, we make a simple calculation: we start with a gas-rich dwarf that has an absolute luminosity of $M_{V}=$ $-16.0 \mathrm{mag}$ and $5 \mathrm{GCs}$, which means $S_{N}=2$ (typical for spirals, e.g. Zepf \& Ashman 1993). We assume that a starburst occurs which involves $10 \%$ of the total mass, of which $20 \%$ will be transformed into clusters. For the duration of the burst this increases $M_{V}$ of the galaxy to about -17.8 mag (assuming that the young stellar population is about $4 \mathrm{mag}$ brighter than a faded old one, Fritze v. Alvensleben \& Burkert 1995). About 12 Gyr after the burst $M_{V}$ has faded again to -16.1 mag. At this time the total luminosity of the clusters is $M_{V}=-11.8$ if no cluster has been destroyed, or $M_{V}=-11.0$ if as many clusters as corresponding to about $50 \%$ of the total cluster light have been destroyed. Adopting for the evolved GCS a typical GCLF (t5-function) with a turnover magnitude of $M_{V, \mathrm{TO}}=$ $-7.4 \mathrm{mag}$ and a dispersion of $\sigma=1.0$ (e.g. Kohle et al. 1996), about 30 or 18 GCs have survived, respectively. The "specific frequency" of the GCS at the time of the starburst is still quite low, $S_{N}=2.7$ for 35 clusters (or cluster candidates), since the galaxy itself is dominated by the young bright stellar light. Such low $S_{N}$ values were determined for Local Group irregulars including the LMC (Harris 1991). After 12 Gyr $S_{N}$ has increased significantly, $S_{N}=8.4$ for 23 GCs (or even $S_{N}=12.7$ for 35 GCs).

If the starburst is 10 times weaker ( $1 \%$ of the total mass), only $3-5$ clusters would have survived and the resulting $S_{N}$ is only slightly larger than before, $S_{N}=$ $3.5 \pm 0.5$. 


\subsection{Estimation of the final $S_{N}$ of a starburst}

Furthermore we want to answer the following question. What is the specific frequency of the starburst itself, without an already existing old stellar population? In other words, we consider an isolated gas cloud and assume that some mechanism has triggered a starburst as strong as observed in starburst galaxies. Then we "destroy" about 20 to $60 \%$ of the cluster light, and look how many GCs survived compared to the total luminosity of the whole system. Note that stars and clusters fade in the same way (according to the models by Fritze v. Alvensleben \& Burkert 1995). The final GCLF has by definition the shape of a t5-function with $M_{V, \text { TO }}=-7.4 \mathrm{mag}$ and $\sigma=1.0$. We assume that immediately after the burst $20 \%$ of the light comes from clusters and that 1000 GCs will survive the evolution. Table 6 summarizes the results. In column 1 the fraction $f_{\text {destr }}$ of GC light that has been disrupted during the evolution is given. Columns 2, 3, and 4 are the absolute luminosities of the evolved GCs, stars, and the total system, respectively. Column 5 gives the resulting $S_{N}$ of the system.

Table 6. Specific frequencies of a starburst, in which 1000 GCs are contained in the final GCLF. The values have been derived under the assumption that $20 \%$ of the initial starburst light comes from clusters (Meurer et al. 1995) and a fraction of the cluster light $f_{\text {destr }}$ has been supplied to the field star light due to cluster destruction

\begin{tabular}{rrrrr}
\hline$f_{\text {destr }}$ & $M_{V, \mathrm{GCs}}$ & $M_{V, \text { stars }}$ & $M_{V, \text { total }}$ & $S_{N}$ \\
\hline 0.2 & -15.6 & -17.4 & -17.6 & 89.0 \\
0.3 & -15.7 & -17.6 & -17.8 & 75.7 \\
0.4 & -15.7 & -17.9 & -18.0 & 62.3 \\
0.5 & -15.7 & -18.1 & -18.2 & 51.1 \\
0.6 & -15.6 & -18.3 & -18.4 & 44.5 \\
\hline
\end{tabular}

The calculations show that $S_{N}$ in an isolated starburst is very high, $40<S_{N}<90$. We note that there exists no evidence that such a high $S_{N}$ can be the result of a simple undisturbed galaxy formation. In particular, dEs, whose structural properties are most easily explained by a starburst followed by a supernova-driven wind (e.g. Dekel \& Silk 1986), have much lower $S_{N}$ values. However, in the context of the galaxy infall scenario, our calculations might imply that stripped gas from galaxies - and especially dwarf galaxies expell their gas most easily (i.e. Dekel \& Silk 1986) - can significantly increase the GC $S_{N}$ of the central GCS, if it suffers a starburst comparable to that observed in starburst galaxies. In Sect. 9 we apply these results to NGC 1399.

\section{Constraints and estimations for the dwarf galaxy infall scenario in the Fornax cluster}

\subsection{Why the present day dwarf galaxy population supports the hypothesis of early infall}

In a scenario where a sufficient number of dwarfs has been dissolved into a $\mathrm{cD}$ halo, one would expect a flat faint end slope of the LF compared to the initial value. López-Cruz et al. (1997) found in a sample of 45 clusters that clusters with a pronounced $\mathrm{cD}$ galaxy indeed tend to have a flat LF faint end slope. This is what we also find for the Fornax cluster.

Furthermore, the surface density slope of $\mathrm{dE}$ and $\mathrm{dS} 0$ galaxies within the core radius of the cluster $\left(r_{\mathrm{c}}=0.7\right)$ is flatter than the slope of all possibly dissolved and/or stripped material: cD halo stars, GCs, and perhaps rest gas. This is consistent with White's (1987) argument that disrupted material is more concentrated than the relaxed galaxy distribution.

Moreover, like in other evolved clusters, the gas-rich late-type Fornax galaxies are found at the outskirts of the cluster, whereas the early-type (possibly stripped) dwarfs are more concentrated towards the center (Ferguson 1989).

One also expects that the LF of compact dwarfs is steeper than the LF of less compact dwarfs (since they are more easily disrupted). Indeed, in the Fornax cluster the LF of the non-nucleated dE/dS0s is flatter than the LF of the (on the average) more luminous nucleated $\mathrm{dE} / \mathrm{dS} 0 \mathrm{~s}$.

Furthermore, if the stripping of gas and stars was more effective in the inner regions, one would expect to find a larger number of fainter remnants in the center than outside. This is indeed seen for the non-nucleated $\mathrm{dE} / \mathrm{dS} 0 \mathrm{~s}$. The fainter dwarfs are more concentrated to the center than the brighter ones (Ferguson \& Sandage 1988).

Finally, two probable candidates for survived nuclei of dissolved dwarfs have been found (see Papers 1 and 2), which would indicate that also dwarfs from the brighter nucleated $\mathrm{dE} / \mathrm{dS} 0$ population have been dissolved.

\subsection{Constraints from the metallicity distribution}

Constraints on the metallicity have to be considered, if one assumes that the metallicity distribution of the GCs is bimodal rather than equally distributed over the range of possible GCs metallicities, $-2.0<[\mathrm{Fe} / \mathrm{H}]<$ 0.0 dex. Primordial gas, expelled and stripped from low mass dwarfs, is normally believed to be a contributor to the metal-poor GC subpopulation $([\mathrm{Fe} / \mathrm{H}]<-1.1 \mathrm{dex})$, if transformed into GCs. However, this may not always be the case. Mac Low \& Ferrara (1999) calculated that galaxies less massive than about $10^{8} M_{\odot}$ can eject metals from supernovae into the intergalactic medium easier than their interstellar gas. Thus, the expelled gas might also be 
enriched from the supernovae ejecta and more metal-rich GCs could have been formed as well. The capture of GCs of early-type dwarf galaxies can only have contributed to the metal-poor GCs, since all observed GCs in such dwarfs seem to be more metal-poor than $[\mathrm{Fe} / \mathrm{H}]=-1.2$ dex (e.g. Minniti et al. 1996). Côté et al. (1998) have shown via Monte Carlo simulations that the capture of dwarf galaxies can indeed reproduce the bimodal color distribution around M 49 and M 87 under the assumption that the red globular cluster population is the intrinsic GCS of the galaxies. The mean metallicity of the captured GCs peaks around $\overline{[\mathrm{Fe} / \mathrm{H}]}=-1.3$ dex for a steep initial $\mathrm{LF}$ slope in their simulations. However, they do not include dwarf galaxies fainter than $M_{V}=-13$ mag. As shown in Sect. 6, the inclusion of these galaxies and an extrapolation of the metallicity-luminosity relation for their GCs, can push the mean metallicity of the captured GCs to a lower value.

Since the metallicity of the accreted stellar population of dwarfs itself is between -2.0 to -0.6 (taking the values of Local Group dwarfs, e.g. Grebel 1997), one should also see a low metallicity in the $\mathrm{cD}$ halo light. Unfortunately, the metallicity determination of the $\mathrm{cD}$ halo is quite difficult due to the low surface brightness, and in the center the light of the bulge of NGC 1399 would dominate a metalpoor halo component. On the other hand, the metallicity of the halo is probably a mixture of different metallicities, if one assumes that not only metal-poor dwarfs have been dissolved, but also stellar populations of more massive galaxies had been stripped and new stars from infalling gas of higher metallicities might have formed.

To explain by the accretion scenario the majority of the red metal-rich $\mathrm{GC}$ subpopulation $([\mathrm{Fe} / \mathrm{H}] \simeq-0.6 \mathrm{dex})$, either already existing GCs of this metallicity had to be captured, or GCs had to be newly formed from enriched infalling gas. This is possible, if one allows that the gasrich dwarfs first had time to enrich their interstellar matter to at least -0.8 dex, before the stripping of the gas became important and/or before new cluster formation in these dwarfs has been triggered by interaction processes. In the LMC, for example, no clusters were formed between about 3 to 10 Gyr ago. Whereas the few old clusters have a metallicity of about $-1.8 \mathrm{dex}$, the younger clusters have metallicities around -0.4 dex (e.g. Olszewski et al. 1991; Hilker et al. 1995). Concerning the time scale for metal enrichment in spirals, e.g. Möller et al. (1997) estimated that $2-3 \mathrm{Gyr}$ is enough time to enrich the iron abundance of the interstellar medium from -1.5 dex to about -0.6 dex for early-type spirals ( $\mathrm{Sa}, \mathrm{Sb}$ ), whereas at least 7 Gyr are needed for late-type spirals (Sc, Sd). Similarly, Fritze-v. Alvensleben \& Gerhard (1994) calculated the metallicity of a secondary GC population in a early-type spiral-spiral merger to be about $[\mathrm{Fe} / \mathrm{H}]=$ -0.6 dex after about 2 Gyr of their life time, and after about 8 Gyr in a late-type spiral-spiral merger.
Assuming that the metal-rich GC population in Fornax was formed by the infall of all types of gas-rich galaxies, one therefore should expect a range of ages among them in order to account for a metallicity peak around -0.6 dex. First, the metal-rich GCs should be at least 2 Gyr younger than the metal-poor GCs with $[\mathrm{Fe} / \mathrm{H}]=$ -1.3 dex. Second, their age spread should be in the order of $2-6$ Gyr. It would be interesting to know whether this prediction can be proved or disproved in further investigations.

We note that a large number of metal-rich GCs also might have been accumulated by stripping from more massive early-type galaxies. According to the metallicityluminosity relation by Côté et al. (1998), the mean metallicity, $[\mathrm{Fe} / \mathrm{H}]=-0.6 \mathrm{dex}$, of the red GC population around NGC 1399 corresponds to a luminosity of the former parent galaxies of about $M_{V}=-20 \mathrm{mag}$. This value is typical for the low-luminosity ellipticals in Fornax, as for example NGC 1374, NGC 1379, and NGC 1427.

\subsection{Constraints from the spatial distribution of globular clusters}

A further point that has to be explained is why the red GC population is more concentrated than the blue one (Forbes et al. 1997). The answer might be that star formation from infalling gas is more concentrated to the inner part of the dense cluster core, as it is also expected for a merging scenario (Ashman \& Zepf 1992). Another possibility is that most of the red GCs have nothing to do with a secondary formation or accretion process, but rather belong to the original GC population of the bulge of NGC 1399. However, as we show in the next section, not more than about 1300 GCs can belong to the bulge light, if one assumes reasonable values of the initial GC specific frequency. This comprises only about half of the red GC subpopulation.

\section{The correct mix of accreted and newly formed GCs}

Let us imagine that the infall of dwarf galaxies and gas was really the dominating process for the building-up of the $\mathrm{cD}$ halo and the GCS. How many dwarfs and their transformed gas would then have contributed to the $\mathrm{cD}$ halo light and how many GCs might belong to the $\mathrm{cD}$ halo?

NGC 1399 possesses about 5800 globular clusters (see Sect. 3.2). About 1300 of them would belong to the bulge, $M_{V, \text { gal }}=-21.5 \mathrm{mag}$ (see Sect. 3.3.1), if one assumes an initial specific frequency of $S_{N}=3.2$, which is the mean value for the other ellipticals in the Fornax cluster, except NGC 1404 and NGC 1380. That means that 4500 GCs would belong to the $\mathrm{cD}$ halo and its specific frequency would be about $S_{N}=10 \pm 1$. Note that half of the total 
Table 7. Possible mixtures of different processes of GC accretion, formation, and stripping that can explain the observed $\mathrm{cD}$ halo properties. In each line, the contribution of each process can be added to the following properties of the $\mathrm{cD}$ halo: $M_{V, \mathrm{cD}}=$ $-21.65 \mathrm{mag}, N_{* \text { tot }, \mathrm{cD}}=4500$, and $S_{N}=10$. See text for more details

\begin{tabular}{crrrrrrrrr}
\hline & \multicolumn{4}{c}{ accretion of GCs } & \multicolumn{1}{c}{ new formation of GCs } & \multicolumn{3}{c}{ stripping of GCs } \\
case & $M_{V}$ & $S_{N}$ & $N_{\mathrm{GC}}$ & $M_{V}$ & $S_{N}$ & $N_{\mathrm{GC}}$ & $M_{V}$ & $S_{N}$ & $N_{\mathrm{GC}}$ \\
\hline 1 & -21.11 & 9.0 & 2500 & -20.63 & 11.2 & 2000 & & & \\
2 & -21.11 & 9.0 & 2500 & -20.53 & 9.2 & 1500 & -18.05 & 30 & 500 \\
3 & -21.52 & 6.2 & 2500 & -19.25 & 40.0 & 2000 & & & \\
4 & -21.59 & 5.8 & 2500 & -18.49 & 80.0 & 2000 & & & \\
5 & -21.52 & 4.9 & 2000 & -18.81 & 60.0 & 2000 & -18.05 & 30 & 500 \\
6 & -21.22 & 4.9 & 1500 & -20.44 & 20.0 & 3000 & & & \\
7 & -21.45 & 3.9 & 1500 & -19.69 & 40.0 & 3000 & & & \\
8 & -21.16 & 3.4 & 1000 & -20.44 & 20.0 & 3000 & -18.05 & 30 & 500 \\
\hline
\end{tabular}

GCS (=2900 GCs) are assigned to the metal-poor peak around $[\mathrm{Fe} / \mathrm{H}] \simeq-1.3 \mathrm{dex}$, and therefore at least 1600 metal-rich $\mathrm{GCs}([\mathrm{Fe} / \mathrm{H}] \simeq-0.6 \mathrm{dex})$ have to be explained by the infall scenario, if one assumes that all 1300 remaining bulge GCs belong to the metal-rich sub-population.

How can dwarf galaxies account for such a high $S_{N}$ ?

As presented in Sect. 5, there are mainly three scenarios possible. Firstly, accreted gas-poor dwarfs possessed high GC frequencies themselves. In this case, the average $S_{N}$ of all accreted dwarfs and GCs can have values between 4 and 22 depending on the initial conditions (see Table 5). Secondly, the infalling gas of previously gasrich dwarfs was effectively converted into globular clusters. Regarding the starburst as an isolated entity its resulting systems of stars and clusters can have $S_{N}$ values between 40 and 90 (see Table 6). Finally, the stripping of GCs from dwarf galaxies was more effective than the stripping of their field population. That this is in principal possible is indicated by the fact that the $S_{N}$ value of the outer parts of galaxies that are primarily affected by stripping can be in the order of 30 (see Sect. 5.1, case 1b).

Among these 3 possibilities the stripping of GCs from dwarf galaxies most probably plays a minor role. Even if all $50 \mathrm{dE} / \mathrm{dS} 0 \mathrm{~s}$ within the core radius of the galaxy distribution are remnants, whose outer GCs have been stripped off, we calculate that maximally some hundred GCs have been captured by this process, assuming an initial $S_{N}=5.5, S_{N}=30$ for the stripped stars and GCs, and a final $S_{N}=3.0$ for the remnant (similar to the values for NGC 4472, McLaughlin et al. 1994). In the following, we consider the case that at most 500 GCs have been stripped.

What is the correct mixture of the two other processes that fulfil the following assumptions?

(1) The cD halo has been formed only by accreted and newly formed matter, and its total luminosity is $M_{V, \mathrm{cD}}=-21.65 \mathrm{mag}$.

(2) The specific frequency of the accreted and newly formed GCs with respect to the halo luminosity is $S_{N}=$ 10, (= $4500 \mathrm{GCs})$.
(3) The 4500 GCs in the cD halo consist of 2500 metalpoor (blue) and 2000 metal-rich (red) GCs (this implies that the GCS of the bulge has 400 metal-poor and 900 metal-rich GCs).

(4) GCs captured by accretion and stripping of dwarfs can only be metal-poor.

In Table 7 we present the possible mixtures of the 3 processes, starting with cases for which GC accretion is dominant and ending with cases in which most GCs have been formed from infalling gas.

In the first five cases we assumed that all metal-poor GCs were captured or stripped. Assuming a high $S_{N}$ value for the accretion process $\left(S_{N}=9\right.$, cases 1 and 2$)$, the cluster formation efficiency (CFE) does not need to be as high as estimated for merger and starburst situations (see Table 6). However, as discussed in Sect. 6, a high $S_{N}$ requires a high accretion rate of dwarf galaxies, a steep initial slope of the faint end of the galaxy LF, and also very faint dwarf galaxies should have possessed at least one GC. The faintest dwarf galaxies with a GCS observed so far are the Local Group dSphs Fornax and Sagittarius $\left(M_{V} \simeq-12.5 \mathrm{mag}\right)$.

The other way around, if starbursts from stripped gas can produce a high $S_{N}$ value $\left(40<S_{N}<80\right.$, cases 35 ) and have formed 2000 metal-rich GCs, the $S_{N}$ for the accreted metal-poor GCs is in the order 5-6. Such values can easily be achieved by the accretion scenario presented in Sect. 6 under various reasonable initial conditions (see Table 5).

In the cases 6 to 8 we assumed that the majority of the GCs had their origin from infalling gas with a low value of the estimated starburst CFEs $\left(20<S_{N}<40\right)$. The specific frequency for the remaining 1500 accreted GCs then can be very low $\left(3<S_{N}<5\right)$, very faint dwarfs do not need to possess GCs, and the numbers of dissolved dwarfs can be of the order of $250-500$. However, one then has to assume that GCs formed from metal-rich as well as metalpoor gas and that most of the original dwarf galaxies have been very gas-rich. 


\section{Concluding remarks}

We have summarized the properties of the different components of the central galaxy NGC 1399 (GCS, cD halo, bulge) and the dwarf galaxy population in the center of the cluster. We have analysed under which circumstances the GCS and cD halo can be explained by the infall and accretion of gas-poor as well as gas-rich dwarf galaxies.

Estimations of the GC formation efficiency from infalling gas, and simulations of the accretion of GCSs from early-type dwarfs have shown that the building-up of the cD halo and central GCS by dwarf galaxy and gas infall alone is only possible under special conditions during the formation and evolution of the cluster. Depending on the leading process which contributed most to the rich GCS, the following conditions are required to fulfil the observed properties:

- There are as many blue (metal-poor) as red (metalrich) GCs seen around NGC 1399. Since not all metalrich GCs can be assigned to the bulge of NGC 1399 when adopting a reasonable $S_{N}$, the formation of secondary metal-rich GCs from stripped gas (or within the dwarf galaxies) probably was an effective process besides the capture of metal-poor GCs.

- The stripping and capture of GCS of gas-poor dwarf galaxies can only account for the metal-poor GC population.

- If the accretion of gas-poor dwarfs was a dominating process, the faint end slope of their initial LF had to be as steep $(\alpha<-1.4)$ as it is predicted in CDM models in order to provide a sufficient number of dwarfs that have been disrupted in the central galaxy.

- A steep faint end slope of the initial LF leads to a mean metallicity of the captured dwarfs that is about 0.5 dex more metal-poor than the observed value of the blue GC population around NGC 1399.

- Furthermore, in the accretion dominated scenario, an unlikely high number of dwarfs $(\simeq 6000$, see Table 5$)$ had to be accreted, and about $50 \%$ of the fainter dwarfs $\left(-12.5<M_{V}<-10.5\right)$ must have possessed at least one GC in order to produce high $S_{N}$ values.

- A very efficient increase in $S_{N}$ of the central GCS by the formation of GCs from gas can be achieved, if the cluster formation efficiency was as high as in merging or starburst galaxies.

- If the majority of the GCs (metal-poor as well as metal-rich ones) formed from stripped gas, a significant fraction of the gas was enriched to at least -1.0 dex before forming GCs in order to explain the bimodal metallicity distribution of the central GCS. This implies that the metal-rich GCs should be at least 2 Gyr older than the metal-poor ones and should show a significant age spread.

Certainly, some of the requirements are quite restrictive. We conclude that the infall of dwarf galaxies can principally explain many properties in the center of the Fornax cluster, but is most probably not the only process that has been active. Certainly, also the brighter, more massive galaxies were envolved by the interaction processes in the central region of the Fornax cluster. A natural extension of the dwarf galaxy infall scenario is, for example, the stripping (and early merging) of giant galaxies - ellipticals and spirals (as mentioned in Sect. 8.2). Besides the low-luminosity ellipticals in Fornax, very likely candidates for stripping are the central giant galaxies NGC 1380 and NGC 1404, which have low GC specific frequencies, and might therefore have provided a significant fraction of the central GCS (see Kissler-Patig et al. 1999).

We are aware of the fact that our proposed scenario has to be tested and confirmed by further theoretical as well as observational work. Especially, it has to be shown in N-body simulations whether the accretion rate of dwarf galaxies can be very high, and what is the dynamical behaviour of stripped and accreted GCs in the central cluster potential. Furthermore, it has to be tested under which conditions a high cluster formation efficiency can be obtained from stripped gas (whether it is comparable to a starburst situation in a galaxy or not). On the observational side it has to be shown, whether the faintest dwarf galaxies possess GCs or not. Further investigation of the faint end slopes of galaxy LFs for clusters with very different properties (redshift, richness, compactness, existence of a cD galaxy, etc.) will show whether the proposed scenario is compatible with the findings. Observations more sensitive to the ages of GCs (i.e. measurement of line indices) will prove or disprove the predictions of an age spread among the GCs.

Acknowledgements. This research was partly supported by the DFG through the Graduiertenkolleg "The Magellanic System and other dwarf galaxies" and through grant Ri 418/5-1 and Ri 418/5-2. MH thanks Fondecyt Chile for support through "Proyecto FONDECYT 3980032" and LI for support through "Proyecto FONDECYT 8970009".

\section{References}

Arnaboldi M., Freeman K.C., Hui X., Capaccioli M., Ford H., 1994, ESO Messenger 76, 40

Ashman K., Zepf S.E., 1992, ApJ 384, 50

Bassino L.P., Muzzio J.C., Rabolli M., 1994, ApJ 431, 634

Bicknell G.V., Carter D., Killeen N., Bruce T., 1989, ApJ 336, 639

Binggeli B., 1994, in: ESO/OHP Workshop on Dwarf Galaxies, Meylan G., Prugniel P. (eds.). ESO, Garching

Binggeli B., Cameron L.M., 1991, A\&A 252, 27

Blanchard A., Valls-Gabaud D., Mamon G.A., 1992, A\&A 264, 365

Bothun G.D., Impey C.D., Malin D.F., 1991, ApJ 376, 404

Brown J.H., Burkert A., Truran J.W., 1995, ApJ 440, 666

Burstein D., Davies R.L., Dressler A., et al., 1984, ApJS 64, 601

Caldwell N.L., Bothun G.D., 1987, AJ 94, 1126 
Cellone S.A., Forte J.C., Geisler D., 1994, ApJS 93, 397

Cellone S.A., Forte J.C., 1996, ApJ 461, 176

Cepa J., Beckman J.E., 1988, A\&A 200, 21

Cole S., 1991, ApJ 367, 45

Cole S., Aragon-Salamanca A., Frenk C.S., et al., 1994, MNRAS 271, 781

Côté P., Marzke R.O., West M.J., 1998, ApJ 501, 554

Da Costa G.S., Armandroff T.E., 1995, AJ 109, 2533

Davies J.I., Phillipps S., Cawson M.G.M., et al., 1988, MNRAS 232,239

Dekel A., Silk J., 1986, ApJ 303, 39

de Vaucouleurs G., de Vaucouleurs A., Corwin H.G., et al., 1991, Third Ref. Catalogue of Bright Galaxies. Springer, New York

Drinkwater M.J., Gregg M.D., Holman B.A., 1997, in ASP Conf. Series, Vol. 116, Proceedings of the Second Stromlo Symposium "The Nature of Elliptical Galaxies" Arnaboldi M., Da Costa G.S. \& Saha P. (eds.)

Durrell P.R., Harris W.E., Geisler D., Pudritz R., 1996, AJ 112,972

Elmegreen B.G., Kaufman M., Thomasson M., 1993, ApJ 412, 90

Evans R., Davies J.I., Phillipps S., 1990, MNRAS 245, 164

Faber S.M., 1973, ApJ 179, 423

Faber S.M., Wegner G., Burstein D., et al., 1989, ApJS 69, 763

Ferguson H.C., 1989, AJ 98, 367

Ferguson H.C., Binggeli B., 1994, A\&AR 6, 67

Ferguson H.C., Sandage A., 1988, AJ 96, 1520

Ferguson H.C., Sandage A., 1991, AJ 101, 756

Ferland G.J., Fabian A.C., Johnstone R.M., 1994, MNRAS 266, 399

Forbes D.A., Brodie J.P., Grillmair C.J., 1997, AJ 113, 1652

Franx M., Illingworth G., Heckman T., 1989, ApJ 344, 613

Fritze-v. Alvensleben U., Burkert A., 1995, A\&A 300, 58

Fritze-v. Alvensleben U., Gerhard O.E., 1994, A\&A 285, 751

Fritze-v. Alvensleben U., Kurth O., 1997, in: proceedings of IAU Symposium 186 "Interacting Galaxies at Low and High Redshift", August 18-22, Kyoto, Japan

Gallagher J.S., Ostriker J.P., 1972, AJ 77, 288

Goudfrooij P., Hansen L., Jørgensen H.E., Nørgaard-Nielsen H.U., de Jong T., van den Hoek L.B., 1994b, A\&AS 104, 179

Gratton R.G., Fusi Pecci F., Carretta E., et al., 1997, in: "Hipparcos Venice'97 Symposium", ESA SP-402

Grebel E.K., 1997, RvMA 10, 29

Grillmair C.J., Forbes D.A., Brodie J.P., Elson R.A.W., 1998, A\&AS 192, 5403

Harris W.E., 1991, ARA\&A 29, 543

Harris W.E., Pudritz R.E., 1994, ApJ 429, 177

Held E.V., Mould J.R., 1994, AJ 107, 1307

Hilker M., Infante L., Vieira G., Kissler-Patig M., Richtler T., 1999, A\&AS 134, 75 (Paper II)

Hilker M., Kissler-Patig M., 1996, A\&A 314, 357

Hilker M., Kissler-Patig M., Richtler, T., Infante L., Quintana H., 1999, A\&AS 134, 59 (Paper I)

Hilker, M., Richtler, T., Gieren, W., 1995, A\&A 294, 648

Hodge P., 1994, in "The Formation and Evolution of Galaxies", V Canary Islands Winter School of Astrophysics, MuñozTuñón C. \& Sánchez F. (eds.). Cambridge, University Press, p. 1

Holtzman J.A., Faber S.M., Shaya E.J., et al., 1992, AJ 103, 691
Ikebe Y., Ezawa H., Fukazawa Y., et al., 1996, Nat 379, 427

Irwin M.J., Davies J.I., Disney M.J., Phillipps S., 1990, MNRAS 245, 289

Jerjen H., Dressler A., 1997, A\&AS 124, 1

Jerjen H., Tammann, G.A., 1997, A\&A 321, 713

Jog C.J., Solomon P.M., 1992, ApJ 387, 152

Jones C., Stern C., Forman W., et al., 1997, ApJ 482, 143

Kauffmann G., Guiderdoni B., White S.D.M., 1994, MNRAS 267,981

Kauffmann G., White S.D.M., Guiderdoni B., 1993, MNRAS 264,201

Killeen N.E.B., Bicknell G.V., 1988, ApJ 325, 165

Kissler-Patig M., Brodie P.B., Schroder L.L., et al., 1998, AJ 115, 105

Kissler-Patig M., Kohle S., Hilker M., et al., 1997, A\&A 319, 470

Kissler-Patig M., Grillmair C.J., Meylan G., et al., 1999, AJ 117,1206

Kohle S., Kissler-Patig M., Hilker M., et al., 1996, A\&A 309, L39

Kormendy J., 1985, ApJ 295, 73

Kroupa P., 1997, New Astron. 2, 139

Lacey C., Guiderdoni B., Rocca-Volmerange B., Silk J., 1993, ApJ 402, 15

Larson R.B., 1993, in "The Globular Cluster Galaxy Connection", ASP Conf. Ser. 48, Smith G.H., Brodie J.P. (eds.), p. 675

Lin D.N.C., Faber S.M., 1983, ApJ 266, L21

Lobo C., Biviano A., Durret F., et al., 1997, A\&A 317, 385

López-Cruz O., Yee H.K.C., Brown J.P., Jones C., Forman W., 1997, ApJ 475, L97

Lutz D., 1991, A\&A 245, 31

Mac Low M.-M., Ferrara A., 1999, ApJ 513, 142

McLaughlin D.E., Harris W.E., Hanes D.A., 1994, ApJ 422, 486

Merritt D., 1984, ApJ 276, 26

Meurer G.R., Heckman T.M., Leitherer C., Kinney A., Robert C., Garnett D.R., 1995, AJ 110, 2665

Miller B.W., Ferguson H.C., Lotz J., Stiavelli M., Whitmore B.C., 1998, ApJ 508, L133

Minniti D., Kissler-Patig M., Goudfrooij P., Meylan G., 1998, AJ 115, 121

Minniti D., Meylan G., Kissler-Patig M., 1996, A\&A 312, 49

Möller C.S., Fritze-v. Alvensleben U., Fricke K.J., 1997, A\&A 317,676

Muzzio J.C., 1987b, PASP 99, 245

Muzzio J.C., Martinez R.E., Rabolli M., 1984, ApJ 285, 7

Nath B.B., Chiba M.S., 1995, ApJ 454, 604

Nørgaard-Nielsen H.U., Goudfrooij P., Jørgensen H.E., Hansen L., 1993, A\&A 279, 61

Nulsen P.E.J., 1982, MNRAS 198, 1007

Okazaki T., Chiba M., Kumai Y., Fujimoto M., 1993, PASJ 45,669

Okazaki T., Tosa M., 1995, MNRAS 274, 48

Olszewski E.W., Schommer R.A., Suntzeff N.B., Harris H.C., 1991, AJ 101, 515

Ostriker J.P., Hausman M.A., 1977, ApJ 217, L125

Ostrov P., Geisler D., Forte J.C., 1993, AJ 105, 1762

Poulain, P., 1988, A\&AS 72, 215

Richstone D.O., 1976, ApJ 204, 642

Richstone D.O., Malumuth E.M., 1983, ApJ 268, 30 
Richtler T., 1995, in "Reviews of Modern Astronomy", Vol. 8, Klare G. (eds.). Springer, p. 163

Sandage A., Binggeli B., Tammann G.A., 1985, AJ 90, 1759

Schechter P., 1976, ApJ 203, 297

Schombert J.M., 1986, ApJS 60, 603

Schombert J.M., 1988, ApJ 328, 475

Schweizer F., Seitzer P., 1993, ApJ 417, L29

Secker J., Harris W.E., 1997, ApJ 469, 623

Silk J., Wyse R.F.G., Shields G.A., 1987, ApJ 322, L59

Stein P., Jerjen H., Federspiel M., 1997, A\&A 327, 952

Thompson L.A., Gregory S.A., 1993, AJ 106, 2197

Trentham N., 1994, Nat 372, 157

Trentham N., 1998, MNRAS 294, 193

Vader J.P., Sandage A., 1991, ApJ 379, L1 van der Marel R.P., 1991, MNRAS 253, 710

Vesperini E., 1997, MNRAS 287, 915

White R.E., 1987, MNRAS 227, 185

White S.D.M., Frenk C.S., 1991, ApJ 379, 52

Whitmore B.C., Schweizer F., Leitherer C., Borne K., Robert C., 1993, AJ 106, 1354

Whitmore B.C., Schweizer F., 1995, AJ 109, 960

Zabludoff A.I., Huchra J.P., Geller M.J., 1990, ApJS 74, 1

Zepf S.E., Ashman K.M., 1993, MNRAS 264, 611

Zinnecker H., Keable C.J., Dunlop J.S., et al., 1988, in "Globular Cluster Systems in Galaxies", IAU Symp. 126, Grinlay J.E. \& Davis Philip A.G. (eds.). Kluwer, Dordrecht, p. 603 